\title{
An Integrated System for Initial Prediction for Autism Spectrum Disorders
}

\author{
K. Ramya, K.M. Sai Kiran, K. Anekh, S. Sathiya Narayanan.
}

\begin{abstract}
Autism Spectrum Disorder ASD specifies where lots of behavioral disabilities, such as social connections and lack of commutating, repetitions of same works, and only single areas are focused. While ASD is noticed in initial stage, some deficiencies and behavioral patterns may not be found as the indication unless they affect a person's life in a particular way. An infrastructure to record, detect and label the behavioural patterns of children with autism spectrum disorder (ASD) has been developed. Autism Spectrum Disorder (ASD) effects the entire life of a person. The major symptoms of ASD are noticed as lack of social contact and communication, same pattern recurrence, predetermined interests and works. The critical issues is ASD identified at initial stage and age. This study, the grouping technique for ASD diagnosis was utilized in children aged 4-11 years. The Linear Discriminant Analysis (LDA) and KNN algorithms are used for categorizing.
\end{abstract}

Keywords: Autism Spectrum Disorder (ASD), Linear Discriminant Analysis (LDA), Feature Selection Techniques (FST)

\section{INTRODUCTION}

There has been an increase in disease and syndromes around the world with each having a different cause and different effect, one such diseases is which is observed from infants to old aged persons, this disorder is developed through neuro and this can be treated, but the prediction for the current disorder is little late in which it makes the patient vulnerable and difficult for him/her to live. The system we have designed helps to recognize the disorder in the early or initial stages for the problem and helps the patient and the family to take necessary steps to cure it and even helps institution to helps students to overcame their difficulty and cope up with peers. We have used Logistic regression algorithm which helps in the categorize the needful symptoms and age group so that the treatment is make quick and the patient resumes back to normal life.

\section{LITERATURE SURVEY}

The system here tells about clinical trails about the drugs and to make it paramount on the monitoring of the effects of the drugs. An in-depth examination of the drugs consequence,

Revised Manuscript Received on April 30, 2020.

* Correspondence Author

K. Ramya*, Assistant professor at SRM Institute of Science and Technology, Ramapuram, Chennai.

K.M.Sai Kiran, Final Year student at SRM Institute of Science and technology, Ramapuram, Chennai.

K. Anekh, Final Year student at SRM Institute of Science and Technology, Ramapuram, Chennai.

Sathiya Narayanan, student at SRM Institute of Science and Technology, Ramapuram, Chennai.

(C) The Authors. Published by Blue Eyes Intelligence Engineering and Sciences Publication (BEIESP). This is an open access article under the CC BY-NC-ND license (http://creativecommons.org/licenses/by-nc-nd/4.0/) side effect and social influences which in turn helps to put a stop to a significant health vandalism. Earlier research explains us that SRS and Electronic health records as an information origin for the outcomes of different cases.

Although both the datasets are not enough to give a unbiased and a complete picture of the patient care and the conditions. In tis study, they have studied, comparing and carefully judge 4 social media platforms, for the data and quality using 11 disease - drug couple. The system has found several affected persons reported on the usage of the serendipitous drugs. They also identified five cases of informal use of English on social media that can be challenging for computers to process, including comparative sentiment, sarcasm, grammar errors, pronoun reference and semantic reference, and emoticons. Their study recommend social media can be supportive latest statistics for the evaluation of medication outcome, and NLP [1]. This paper explains about the usage of drugs that tells us about drugs we use in unknown way as it was intended for another purpose and the drugs gives a relief for the both issues. In the past drugs finding, serendipity has accounted for new and victorious indication for other and innovative ways of usage. The earlier reports say about the drugs utilized in social media. If any potential information has been discovered in the media , it could be helpful for giving rise to a Drug-Repositioning hypotheses. This study framed an observation of drugs usage in social media as a categorization and inquiry on deep neural networks. They constructed a word-embedded quality using word2vec algorithm. We adopted the convolutional neural network (CNN), long short-term memory network (LSTM), and convolutional long short-term memory network (CLSTM) and redesigned them by adding contextual information that we extracted from drug-review posts, information filtering tools, medical ontology, and medical knowledge. We trained, tuned, and evaluated our deep neural network models on a gold standard dataset containing 15,714 sentences, of which 447 contained serendipitous drug usages. The end outcome portrayed factors for non-balanced info sets to lower false positive rate. However, deep neural network models could more effectively utilize word embedding in feature construction. This advantage made deep neural networks worthy of further investigation and improvement [2]. This paper explains about how to address the precision, sparsity, fluke issues of the top items with Collaborative Filtering (CF). Earlier studies show that usual items or import some data to increase the efficiency of CF. In this system developed is towards a big number of unrated items. By usage of serendipity items, we can Concise Satisfaction and Interest Injection (CSII), a technique utilized for finding the interesting, satisfactory items to the user. Here by stopping uninterested items in the top list, this concise but-novel method increases the quality.

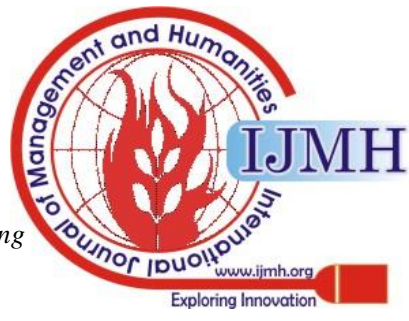




\section{An Integrated System for initial Prediction for Autism Spectrum Disorders}

Meanwhile, it can address the sparsity and cold-start issues by enriching the rating matrix in CF without additional information.

As our method tackles rating matrix before recommendation procedure, it can be applied to most existing CF methods, such as item-based CF, user-based CF and Matrix Factorization-based CF. Through a intensive trail using the huge data on real world datasets, we can reveal a answer to the performance of the old CF methods universally. Therefore, contrasting our baseline techniques, CSII can distill the unwanted items with lot of precaution, avoiding the capable items inferred by mistake[3]. Recommender system is $\mathrm{S} / \mathrm{w}$ constructed for the reviving the related data by the analysis on the searched interest. The system will determine the person's interest based on the search history of the resources the person has consumed, looked upon or saw it for long time. This huge value of data regarding the person and the items grouped with intensive research which growth in accuracy of system results in "Over specialization" drawback. These obvious recommendations tend to impact negative on user on his/her long-time interests. For example, a high accuracy travel recommender system will never recommend new places to a user outside of the already visited places. To mitigate this problem, researchers shifted from focusing on accuracy to achieve user satisfaction to a more user central measures known as beyond accuracy measures. These measures believed to increase user satisfaction, and long-term interest [4]. Learning Management System (LMS) is a new age instrument for professors and teachers in making students learn. The milleanls now focus on the internet materials rather than class notes and textbooks and grasp the required information through the slides, pictures and the documents posted by teachers. Since there is an enormous knowledge in present on the internet and the accessibility to it is even faster and efficient students learn faster. In this system we have proposed an algorithm and an application which will categorize, collect and recommend the needful videos from YouTube or other sources to the children based on the content on LMS. We named the application as Video Recommender System. This contains two parts; API and Document processor. System utilizes Term Frequency-Inverse Document Frequency to map the needful information based on the given data. At last system compares the result of the algorithm to one from direct search. [5].

\section{EXISTING SYSTEM}

ASD could also be named as a collection of Neuro disabilities which aren't curable but can be prevented in the early stages. The prevailing system has the information present consist of missing, unnecessary data which are replaced by values. In addition to that various categorical traits which are broken down by the integer values. The collected information concerning infants, adults, adolescents and children used FTs techniques like Z-score, log and sine functions. Many classifiers were used in the change of the data collected and were identified through ML/AI methods. Therefore, different FT procedure were to low down the equality and linear additive relationship of the ASD information.

\section{A. Disadvantages of Existing System \\ - It supports only numerical datasets \\ - Sensitive to distance}

- Sensitive to Outliers

- Not compatible to other analysis

- High Computational Cost

\section{PROPOSED SYSTEM}

The ML method which is used in the separation of two set of the information: train and test datas. Train set comprise label information. Test data comprise the new information which weren't exist in train set. Moreover, Test information cover labels which is used for the examination of the outcomes. The procedures won't compare model that the AUROC which helps to compute the true correct value to its false correct value throughout the various class of threshold. Many medical examined tests are corrected by their specify and sensitivity. Sensitivity is similar as true correct value and specify is 1 minus false positive value.

\section{A. Advantages of Proposed system}

- High Accuracy

- High Performance with respect to large dataset

- Low computation cost

- High Efficiency

- It does not assume a linear relationship

- It may handle nonlinear effects

\section{ARCHITECHTURE DIAGRAM.}

The given diagram tells us the travers of the data and the evaluation of the given dataset and how the actual value or the prediction is calculated and expressed to us in form of formatted outcome or result. Each and every value is taken into understanding and is examined which in turn tells us the exact or approx. behavior of the subject we have taken into consideration.

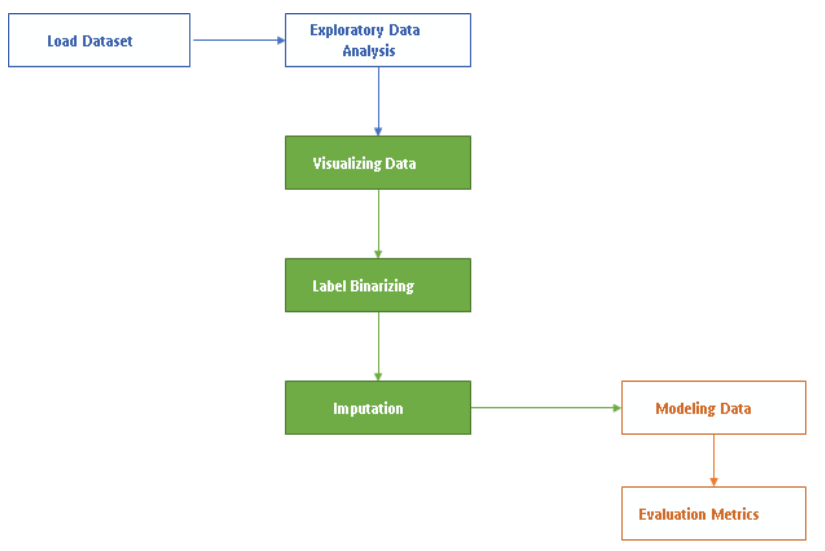

\section{PROJECT MODULES.}

In this project we have three modules which explain the working of the system and gives and in-depth clarity for the working of the system.

\section{A. Module 1: Exploratory Data Analysis.}

EDA informs to the crucial operation of execute pre-inquiry on the information so as to discover patterns, to spot the abnormality, to test and to check premise with help of statistics and graphical presentation.
Published By:

\& Sciences Publication

(C) Copyright: All rights reserved.

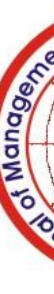

$$
\text { and }
$$$$
\text { and } \mathrm{Hu}_{\mathrm{n}}
$$ 
To use a linear regression for modelling, it needed for detach related values for the betterment of system. Anyone can find the relations utilizing pandas ".corr()" function and can envision the matrix using heatmap in seaborn.

Exploratory Data Analysis (EDA) is an approach/philosophy for data analysis that employs a variety of techniques (mostly graphical) to:

- maximize insight into a data set;

- uncover underlying structure;

- extract important variables;

- detect outliers and anomalies;

- test underlying assumptions;

- develop parsimonious models; and

- determine optimal factor settings.

\section{B. Module 2: Binarizing Labels}

Binarize labels is a 1vsALL method. Many binary classification algorithms can be used for scikit. An easy way for the expansion of these techniques to a multi class case is for usage the 1vsall scheme. This simply has a learner one regressor or binary per class.

LabelBinarizer is a function which helps in transforming the hard process to easier way. At the prediction time, one gives a class to the corresponding model gives the bigger confidence. LabelBinarizer makes reverse transform method for the preparation of easier process.

\section{Module 3: Preparing Data for Model}

Logistic regression may be a statistical procedure for predicting binary classes. the result or target variable is dichotomous in nature. Dichotomous means there are only two possible classes. for instance, it is often used for cancer detection problems. It computes the probability of an occasion occurrence. it's a special case of rectilinear regression where the target variable is categorical in nature. It uses a log of odds because the variable. Logistic Regression predicts the probability of occurrence of a binary event utilizing a logic function.

\section{CONCLUSION}

The system developed has the dataset which was used to apply many ML techniques. The main point is calculating exact value for many methods for a better analysis, then to create a module which can be utilized for ASD prediction.

\section{REFERENCES}

1. Boshu Ru, Kimberly Harris, Lixia Yao "A Content Analysis of Patient-Reported Medication Outcomes on Social Media", October 2015.

2. Dingcheng Li, Lixia Yao “ Detecting Serendipitous Drug Usage in Social Media with Deep Neural Network Models", November 2018

3. Yongjian Yang, Yuanbo Xu, En Wang, Jiayu Han;,Zhiwen Yu "Improving Existing Collaborative Filtering Recommendations via Serendipity-based Algorithm”, August 2018.

4. Fakhri Abbas "Serendipity in Recommender System: A Holistic Overview", October 2018.

5. Natthanun Chantanurak, Proadpran Punyabukkana, Atiwong Suchato "Video Recommender System using textual data: Its Application on LMS and Serendipity Evaluation”, November 2018.

6. Tania Akter, MD. Shahriare Satu, MD. Imran Khan "Machine Learning-Based Models for Early Stage Detection of Autism Spectrum Disorders", 2016.

\section{AUTHORS PROFILE}

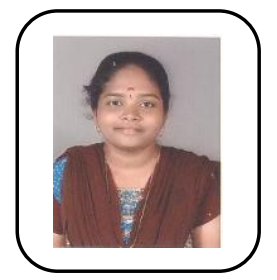

K. Ramya., Assistant professor at SRM Institute of Science and Technology, Ramapuram, Chennai. As a part of curriculum been in head and guide of projects developed by students.

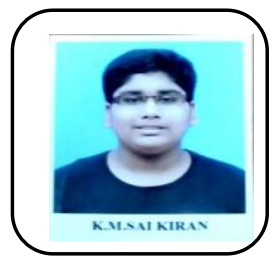

K.m.sai Kiran, Final Year student at SRM Institute of Science and technology, Ramapuram, Chennai.

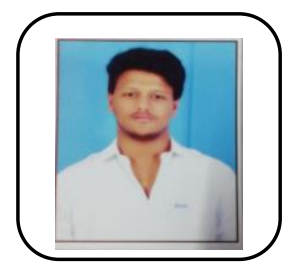

K. Anekh, Final Year student at SRM Institute of Science and Technology, Ramapuram, Chennai. 